\title{
Two dimensional modeling of helical structures, an application to simple strands
}

\author{
Nikolaos Karathanasopoulos ${ }^{\mathrm{a}, *}$, Gerald Kress ${ }^{\mathrm{b}}$ \\ ${ }^{a}$ Institute for Mechanical Systems, ETH Zürich, Leonhardstrasse 21, CH-8092 Zürich, \\ Switzerland, phone: +4144633 6331; fax: +41446321145 \\ ${ }^{b}$ Laboratory of Composite Materials and Adaptive Structures/IDMF, ETH Zürich, \\ Tannenstrasse 3, CH-8092 Zürich, Switzerland
}

\begin{abstract}
Herein, we elaborate a generic, planar finite element model for the simulation of the mechanical response of helical structures to axial and torsional strain. We verify the modeling approach over a wide range of helix angles using closedform expressions. Thereafter, we present a case study application addressing the structural response of single layer engineering strands. Finally, we analyze the model's computational merits.
\end{abstract}

Keywords: Helix, Finite element method, Axial strain, Torsional strain

\section{Introduction}

Helical structures are encountered in nature (e.g. collagen) as well as in numerous engineering applications with ropes, lifting or power line cables and tissue engineering scaffolds being indicative examples.

The mechanical response of helical constructions has been primarily characterized by means of closed-form analytical expressions, while it is only in the last few decades that the notable development of numerical methods has provided new engineering tools for their analysis and design. Nevertheless, the modeling techniques appearing in the literature have been mainly based on beam or volume elements. The latter quickly incur a high number of degrees of freedom (dofs), which respectively entail a significant computational power cost.

Most of the analytical models appearing in the literature based their formulation on thin beam theory considerations. In particular, early works characterized the helix stiffness by taking into account the response contribution arising from the helix cross section axial stiffness EA, as the work of Hruska indicates [1, 2]. Subsequent works, emphasized on the role of the helix cross section torsional

\footnotetext{
* Corresponding author

Email addresses: nkaratha@ethz.ch (Nikolaos Karathanasopoulos), gkress@ethz.ch (Gerald Kress)
}

Preprint submitted to Elsevier

July 31, 2015

(C) 2015. This manuscript version is made available under the Elsevier user license http://www.elsevier.com/open-access/userlicense/1.0/ 
stiffness $G J$, incorporating the corresponding contribution in the provided helix closed-form stiffness expressions [3, 4]. Later on, Costello presented a linearized theory that was based on three generalized strain parameters accounting for helix bending as an additive response mechanism to the above broached ones [5]. To that extent, the variations of the helix curvature, twist and lay angle were taken into account. Velinsky studied the necessity of resorting to non-linear formulations for the helix structural response to be sufficiently characterized [6], concluding that for service strains, the omission of higher order contributions is utterly justified. Furthermore, Raoof and Kraincanic [7] conducted theoretic parametric studies and used experimental data to assess the range of validity of thin rod and orthotropic sheet theory based models, as a function of the helical strand size and geometric configuration. Moreover, Utting et Jones [8, 9] presented a beam theory based model along with a large set of experimental data, while Sathikh et al. [10] were the first to provide a model that complied with the Betti-Maxwell symmetry principle for the two-times-two linearized structural stiffness matrix describing the mechanical response of helices to axial and torsional strain. Their derivations were grounded on a four generalized strain model provided by Ramsey [11].

On the numerical modeling side, most of the presented numerical models were based on volume or beam elements. In particular, Jiang et al. [12, 13, 14] used a strand slice to simulate the response of helical bodies to axial strain, making use of standard volume elements. Nawrocki and Labrosse [15] emphasized on the effect that the helix kinematic considerations have on the stiffness terms of single layer helical strands. Cartraud and Messager [16] applied homogenization theory to beams with periodic micro-structures to simulate the response of helical bodies, applying their modeling approach to single layer engineering strands, while Messager and Cartraud [17] presented a modeling technique based on volumetric finite elements. In more recent works, Usabiaga and Pagalday [18] introduced a modeling approach for the simulation of axial and torsional helix loading, while Ghoreishi et al. [19] pointed out limitations of several analytical schemes. Finally, the impact of kinematic constraints arising from the braiding pattern of multilayer helical assemblies was elaborated, providing bounds for the construction's stiffness [20].

While a large number of volume or beam element based modeling schemes of helical structures has been presented, planar modeling techniques have only recently attracted researcher's attention. In particular, Treysséde presented a SAFE method extension to helical waveguides where the propagation of elastic waves was addressed makingg use of a helix plane [21]. Thereupon, Frikha et al. simulated the structural response of a single layer helical strand to axial straining, grounding their formulation on a helix special twisted basis [22].

\subsection{Structure of the present work}

The present work is based upon Karathanasopoulos et al. [23], with the current work extending the modeling approach to account for torsional strains. What is more, an application to single layer engineering strands is provided, along with a quantification of the computational cost of the numerical model. 
More specifically, in the current work we introduce a generic scheme for the planar finite element modeling of helical structures in Section 2, using a kinematically based method for the loading description. Thereupon, we compare the numerically retrieved structural response for a single helical body to closed-form analytical expressions over a wide range of helix angles for validation purposes. In Section 3, we present an application to simple engineering strands. Thereafter, we discuss on the model's computational merits (Section 4) and conclude in Section 5. 


\section{Model development}

\subsection{Helix geometry}

The three dimensional helix geometry is described making use of the $\mathbf{n}, \mathbf{b}$, t Serret-Frenet basis:

$$
\mathbf{X}\left(x_{n}, x_{b}, s\right)=\mathbf{R}(s)+x_{n} \mathbf{n}+x_{b} \mathbf{b}, \quad-r \leq x_{n}, x_{b} \leq r
$$

where $r$ denotes the radius of the helical body and $\mathbf{R}(\mathrm{s})$ stands for the position vector of the helix centerline, defined as follows:

$$
\mathbf{R}=\left\{\begin{array}{c}
a \cos \varphi \\
a \sin \varphi \\
b \varphi
\end{array}\right\} \quad \varphi=\frac{s}{\gamma} \quad \gamma=\sqrt{a^{2}+b^{2}} \quad \theta=\arctan \left(\frac{b}{a}\right)
$$

In Eq. 2, $a$ and $b$ are intrinsic helix parameters, namely the centerline position of the helix and the helix rise along its axis per unit helix angular evolution $\varphi$. The latter define the curvature $\kappa$ and tortuosity $\tau$ of the helical structure:

$$
\kappa=\frac{a}{\gamma^{2}} \quad \tau=\frac{b}{\gamma^{2}}
$$

The general basis of Eq. 1 is elaborated in Appendix A. On the left side of Fig. 1, a $3 D$ helix reconstruction is provided, while on its right part, a two dimensional helix cross section mesh is depicted.

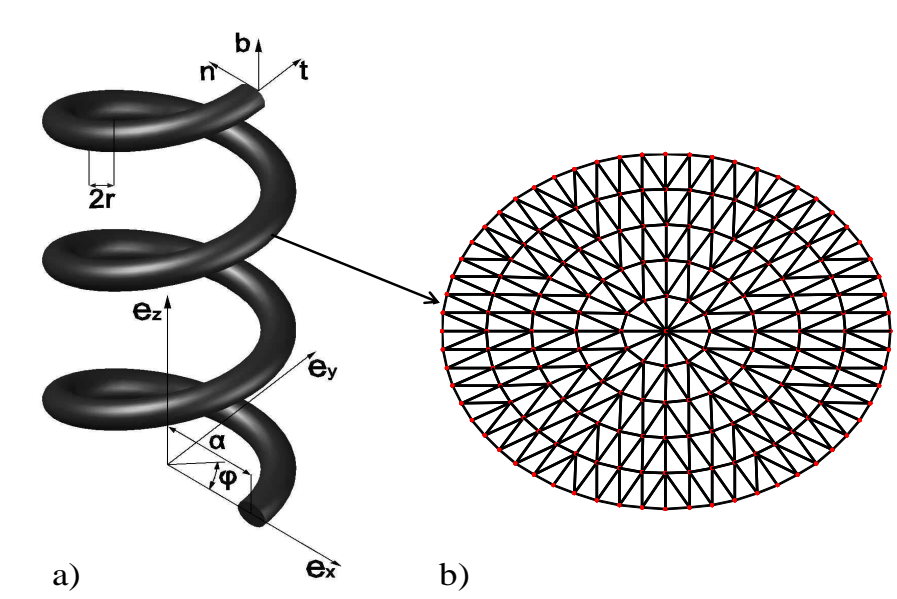

Figure 1: a) Helix and coordinate conventions b) Helix cross section mesh 


\subsection{Kinematic and constitutive equations}

All mechanical formulations are described in a general curvilinear basis, for which the linearized kinematic equations are defined in index or in matrix notation as follows [24]:

$$
\epsilon_{i j}=\frac{1}{2}\left(\left.u_{i}\right|_{j}+\left.u_{j}\right|_{i}\right)=\frac{1}{2}\left(u_{i, j}+u_{j, i}\right)-u_{k} \Gamma_{i j}^{k}, \quad \boldsymbol{\epsilon}=\left(\mathbf{L}_{12}+\mathbf{L}_{3}\right) \mathbf{u}_{123}
$$

where the notation $(1,2,3)$ has been used for the derivation with respect to $\mathrm{x}_{n}$, $\mathrm{x}_{b}$ and $\mathrm{s}$ respectively, while the expansion of the linear operators $\mathbf{L}_{12}$ and $\mathbf{L}_{3}$ is provided in Appendix B. The linear elastic constitutive law is defined as [25]:

$$
\sigma^{i j}=C^{i j k l} \epsilon_{k l}
$$

with superscripts and subscripts denoting respectively contravariant and covariant base components, Appendix A. The stress tensor is defined in vector notation as $\left[\sigma^{11} \sigma^{22} \sigma^{33} \sigma^{23} \sigma^{13} \sigma^{12}\right]^{T}$ while the strain tensor is given as $\left[\epsilon^{11} \epsilon^{22} \epsilon^{33} 2 \epsilon^{23} 2 \epsilon^{13} 2 \epsilon^{12}\right]^{T}$. Finally, the elasticity tensor $\mathbf{C}$ for an elastic and isotropic material is expressed as follows [26]:

$$
C^{i j k l}=\frac{\nu E}{(1+\nu)(1-2 \nu)} g^{i j} g^{k l}+\frac{E}{2(1+\nu)}\left(g^{i k} g^{j l}+g^{i l} g^{j k}\right)
$$

where $E$ and $\nu$ stand respectively for the Young modulus and for the Poisson ratio. The definition of the contravariant metric tensor $\left[g^{i j}\right]$ entering the elasticity tensor is elaborated in Appendix A.1.

\subsection{Displacement field and strain definition}

The planar model simulates the response of the helical body by defining its loading upon the concept of macro strains. Macro strains are described in the usual Cartesian reference basis. Related global deformations are expressed in terms of the applied macro strains $\overline{\boldsymbol{\epsilon}}_{x y z}$. The macro strains are compatible with the displacement field $\overline{\mathbf{u}}_{x y z}$. The total solution of a specified problem consists of two parts:

$$
\mathbf{u}_{x y z}=\mathbf{u}_{x y z}^{F E M}+\overline{\mathbf{u}}_{x y z}
$$

namely the finite element solution part $\mathbf{u}_{x y z}^{F E M}$ and the prescribed displacement field part $\overline{\mathbf{u}}_{x y z}$, while the definition of Eq. 7 can be accordingly viewed in its Curvilinear mapping as follows:

$$
\mathbf{u}_{123}=\mathbf{u}_{123}^{F E M}+\overline{\mathbf{u}}_{123}
$$

Implementing the kinematic relations of Eq. 4 in Eq. 8, the following expression for the mechanical strains is retrieved:

$$
\boldsymbol{\epsilon}_{123}^{M}=\boldsymbol{\epsilon}_{123}^{F E M}+\overline{\boldsymbol{\epsilon}}_{123}
$$




\subsection{Variational formulation}

We elaborate a variational formulation form, starting from the total potential energy $\Pi$ of a linear elastic system, composed of the deformation energy $U$ and of the externally applied work $W$, as follows:

$$
\begin{aligned}
\Pi & =U-W \\
& =\frac{1}{2} \int_{\Omega} \boldsymbol{\epsilon}^{M^{T}} \mathbf{C} \boldsymbol{\epsilon}^{M} d \Omega-\int_{\Gamma_{\sigma}} \mathbf{u}^{T} \hat{\boldsymbol{\sigma}} d \Omega-\int_{\Gamma_{u}} \hat{\mathbf{u}}^{T} \boldsymbol{\sigma} d \Omega,
\end{aligned}
$$

In the above introduced total potential, the external work arises from the prescribed stresses $\hat{\boldsymbol{\sigma}}$ and displacements $\hat{\mathbf{u}}$ on the respective domain boundaries as well as from the prescribed macro strains contributing to the volume integral. Discretizing the domain upon element-wise defined shape functions $\boldsymbol{\Phi}$, the following expression for the mechanical strains is obtained:

$$
\begin{aligned}
\boldsymbol{\epsilon}_{123}^{M} & =\left(\mathbf{L}_{12}+\mathbf{L}_{3}\right) \mathbf{u}_{123} \\
& =\mathbf{L}_{12} \boldsymbol{\Phi}^{T} \tilde{\mathbf{u}}_{123}+\overline{\boldsymbol{\epsilon}}_{123}=\mathbf{B} \tilde{\mathbf{u}}_{123}+\overline{\boldsymbol{\epsilon}}_{123}
\end{aligned}
$$

Where the analytical part of Eq. 7, describing the externally applied strains is elaborated in Section 2.5. By substituting the above expression in the energy potential $\Pi$ of Eq. 10 and by letting its variation with respect to the solution parameters $\overline{\mathbf{u}}_{123}$ vanish, the following system of equations arises:

$$
\mathbf{K} \tilde{\mathbf{u}}_{123}=\mathbf{r}, \quad \mathbf{K}=\sum_{k=1}^{N} \int_{\Omega_{k}} \mathbf{B}^{T} \mathbf{C B} d \Omega_{k}
$$

where the right-hand side, or force vector, $\mathbf{r}$ is given as follows:

$$
\mathbf{r}=-\sum_{k=1}^{N} \int_{\Omega_{k}} \mathbf{B}^{T} \mathbf{C} \overline{\boldsymbol{\epsilon}}_{123} d \Omega_{k}+\sum_{k=1}^{N} \int_{\Gamma_{\sigma_{k}}} \boldsymbol{\Phi}^{T} \hat{\boldsymbol{\sigma}} d \Gamma_{k}
$$

The formulation of Eq. 12 allows for the specification of different mechanical strains. We hereby concentrate on the proposal and verification of axial and torsional strain, elaborated in Section 3.2.

\subsection{Planar mesh load application scheme}

\subsubsection{Axial strain}

We prescribe a homogeneous axial strain field $\bar{\epsilon}_{z}$ along the global Cartesian Z axis, defined through the centerline of the helical body. The latter is compatible with the following displacement field:

$$
\overline{\mathbf{u}}_{x y z}=\left\{\begin{array}{c}
\bar{u}_{x} \\
\bar{u}_{y} \\
\bar{u}_{z}
\end{array}\right\}=\bar{\epsilon}_{z}\left\{\begin{array}{c}
0 \\
0 \\
z
\end{array}\right\}=\frac{b s \bar{\epsilon}_{z}}{\gamma}\left\{\begin{array}{c}
0 \\
0 \\
1
\end{array}\right\}
$$


Computing the local curvilinear displacement components along with the respective displacement gradients - for a transformation angle $\varphi=0$ in Eq. A.10 - we retrieve the following:

$$
\overline{\mathbf{u}}_{123}=\tau s\left\{\begin{array}{l}
0 \\
a \\
b
\end{array}\right\} \bar{\epsilon}_{z}, \quad \nabla \overline{\mathbf{u}}_{123}=\tau\left[\begin{array}{ccc}
0 & 0 & 0 \\
0 & 0 & a \\
0 & 0 & b
\end{array}\right] \bar{\epsilon}_{z}
$$

Evaluation of the kinematic Eq. 4 provides the following applied strain components:

$$
\bar{\epsilon}_{123}=\left[\begin{array}{llllll}
0 & 0 & b \tau & a \tau & 0 & 0
\end{array}\right]^{T} \bar{\epsilon}_{z}
$$

\subsubsection{Torsional strain}

We consider a homogeneous helix twist $\omega^{\prime}$, defining the helix rotation per unit helix development length $L_{z}=b s / \gamma$, so that the absolute rotation $\omega$ increases linearly with the local curvilinear coordinate $s$, as follows:

$$
\omega(s)=\omega^{\prime} \frac{b}{\gamma} s
$$

The applied torsional strain is compatible with the following applied displacement field:

$$
\overline{\mathbf{u}}_{x y z}=\left\{\begin{array}{c}
\bar{u}_{x} \\
\bar{u}_{y} \\
\bar{u}_{z}
\end{array}\right\}=\frac{b}{\gamma} s\left\{\begin{array}{r}
-Y \\
X \\
0
\end{array}\right\} \omega^{\prime}
$$

The curvilinear displacement components - for a transformation angle $\varphi=0$ in Eq. A.10- are thereupon computed as follows:

$$
\overline{\mathbf{u}}_{123}=\tau s\left\{\begin{array}{c}
-b x_{b} \\
-b\left(a-x_{n}\right) \\
b \tau x_{b}^{2}+\left(a-x_{n}\right)^{2}
\end{array}\right\} \omega^{\prime}
$$

with the displacement gradients given as:

$$
\nabla \overline{\mathbf{u}}_{123}=\tau\left[\begin{array}{ccc}
0 & -b s & -b x_{b} \\
b s & 0 & -b x_{n}^{a} \\
-2 x_{n}^{a} s & 2 b s \tau x_{b} & b \tau x_{b}^{2}+\left(x_{n}^{a}\right)^{2}
\end{array}\right] \omega^{\prime}
$$

where in Eq. 20, the abbreviation $x_{n}^{a}=a-x_{n}$ has been used. Evaluating the kinematic Eq. 4 yields the following imposed total strain field expression:

$$
\overline{\boldsymbol{\epsilon}}_{123}^{T}=\left\{\begin{array}{c}
0 \\
0 \\
\tau\left(b \tau x_{b}^{2}+\left(a-x_{n}\right)^{2}\right) \\
-\tau b\left(a-x_{n}\right) \\
-\tau b x_{b} \\
0
\end{array}\right\} \omega^{\prime}
$$




\section{Verification}

\subsection{Analytical closed-form solutions}

We model the helix axial and torsional strain structural response considering the global force and moment resultants $F_{z}$ and $M_{z}$, related through the following system of equations:

$$
\left[\begin{array}{c}
F_{z} \\
M_{z}
\end{array}\right]=\left[\begin{array}{ll}
\kappa_{\epsilon_{z} \epsilon_{z}} & \kappa_{\epsilon_{z} \omega^{\prime}} \\
\kappa_{\omega^{\prime} \epsilon_{z}} & \kappa_{\omega^{\prime} \omega^{\prime}}
\end{array}\right]\left[\begin{array}{c}
\bar{\epsilon}_{z} \\
\omega^{\prime}
\end{array}\right]
$$

We subsequently cite the closed-form expressions provided by Sathikh et al. [10], characterizing the stiffness term expressions of Eq. 22 as functions of the extensional, torsional and bending helix cross section stiffness, EA, GJ and $E I$ respectively:

$$
\begin{aligned}
\kappa_{\epsilon_{z} \epsilon_{z}} & =E A s^{3}+\left(G J c^{2}+E I s^{2}\right) c^{4} s / a^{2} \\
\kappa_{\omega^{\prime} \epsilon_{z}} & =E A a s^{2} c+\left[G J s^{2}-E I\left(1+s^{2}\right)\right] s^{2} c^{3} / a \\
\kappa_{\omega^{\prime} \omega^{\prime}} & =E A a^{2} c^{2} s+E I c^{2} s\left(1+s^{2}\right)^{2}+G J s^{7}
\end{aligned}
$$

where the abbreviations $\mathrm{s}=\sin \theta$ and $\mathrm{c}=\cos \theta$ have been employed, $\theta$ being the helix angle.

\subsection{Planar modeling verification}

A normalized form of the stiffness expressions is employed for the terms comparison so that a non-dimensional form arises, the normalization carried out as follows:

$$
\kappa_{\epsilon_{z} \epsilon_{z}}^{*}=\frac{F_{z}}{\bar{\epsilon}_{z} E A}, \quad \kappa_{\omega^{\prime} \epsilon_{z}}^{*}=\frac{M_{z}}{\bar{\epsilon}_{z} E A a}, \quad \kappa^{*} \omega^{\prime} \omega^{\prime}=\frac{M_{z}}{\omega^{\prime} E A a^{2}}
$$

In Fig. 2 the numerically retrieved axial, coupling and torsional stiffness terms are compared with the closed form solutions of Eq. 23 over the entire range of helical angles. For the verification, a helix index of $a / r=10(\mathrm{r}=0.1)$ has been employed and a Poisson ratio of $\nu=0.3$. 

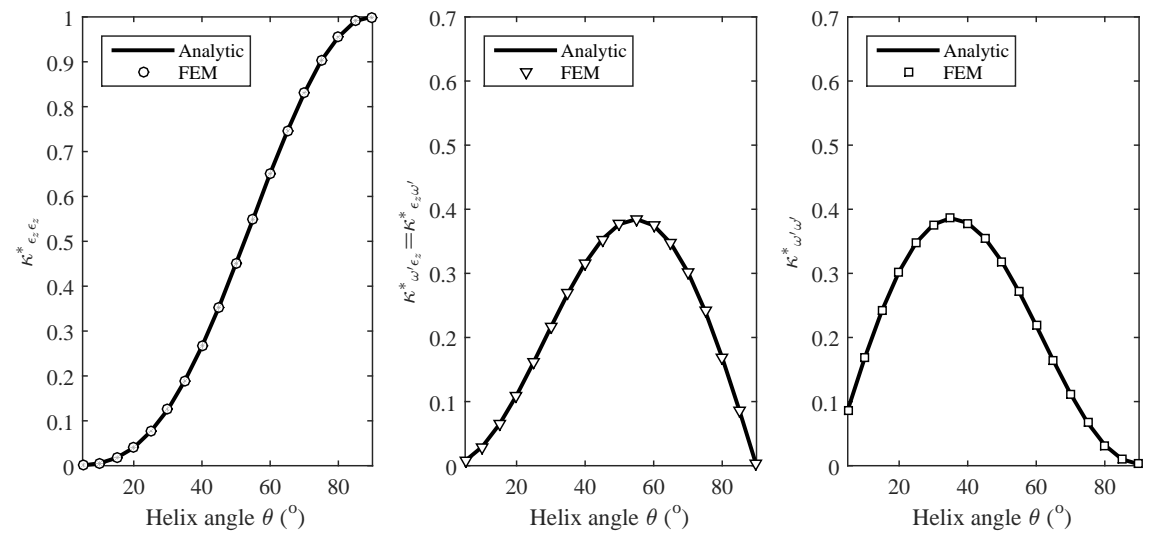

Figure 2: Stiffness terms comparison

The results verify the accuracy of the modeling approach for all stiffness terms, any relevant discrepancies lying below $0.5 \%$.

\subsection{Application to simple helical strands}

The model is subsequently used to simulate the structural response of a single layer engineering strand, its layer comprised of 6 helical bodies. Table 1 summarizes the material and geometric specifications of the construction:

Table 1: Simple strand material and geometric properties

\begin{tabular}{c|c|c|c|c}
\hline & $E\left(\mathrm{KN} / \mathrm{mm}^{2}\right)$ & $r(\mathrm{~mm})$ & $\nu(-)$ & $\theta\left(^{\circ}\right)$ \\
\hline Core $(c)$ & 197.9 & 1.970 & 0.30 & 90.0 \\
Helix $(h)$ & 197.9 & 1.865 & 0.30 & $80.8|77.8| 73 \mid 70$ \\
\hline
\end{tabular}

The modeling results are compared to the ones provided by analytical [10] and numerical modeling schemes [19,22]. Fig. 3 depicts the normalized stiffness term predictions for the axial $\kappa_{\epsilon_{z} \epsilon_{z}}^{\prime}$, coupling $\kappa^{\prime}{ }_{\omega^{\prime} \epsilon_{z}}$ and torsional stiffness $\kappa^{\prime}{ }_{\omega^{\prime} \omega^{\prime}}$ of the helical strand (Table 1) - the normalization carried out with respect to $A_{c}+6 A_{h}, A=6 A_{h} a$ and $6 E A_{h} a^{2}+G J_{c}$ accordingly -. 

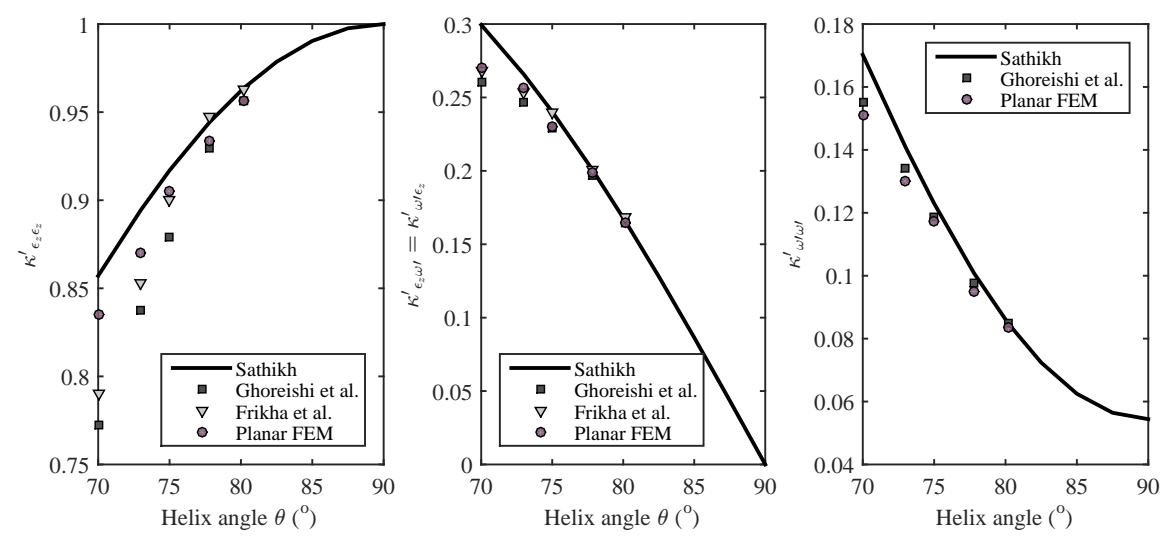

Figure 3: Simple helical strand stiffness comparison

Fig. 3 indicates that all modeling predictions are in very good agreement for steep helix angles $\left(\theta=80.8 \mid 77.8^{\circ}\right)$, with their maximum relative difference being below $2 \%$. Nevertheless, the lower the helix angle that the helical layer follows is $\left(\theta=73.8 \mid 70^{\circ}\right)$, the more compliant the numerically retrieved response is, with analytical closed-form solutions overestimating the constructions' stiffness. In particular, the purely axial stiffness $\kappa^{\prime} \epsilon_{z} \epsilon_{z}$ is overestimated by a maximum of $9 \%$ for a strand with a helix layer angle of $70^{\circ}$, as the comparison with the volume finite element prediction suggests.

Fig. 4 below depicts the normal stress distribution of the strand when subject to axial strain, for a helical layer angle of $80.8^{\circ}$ :

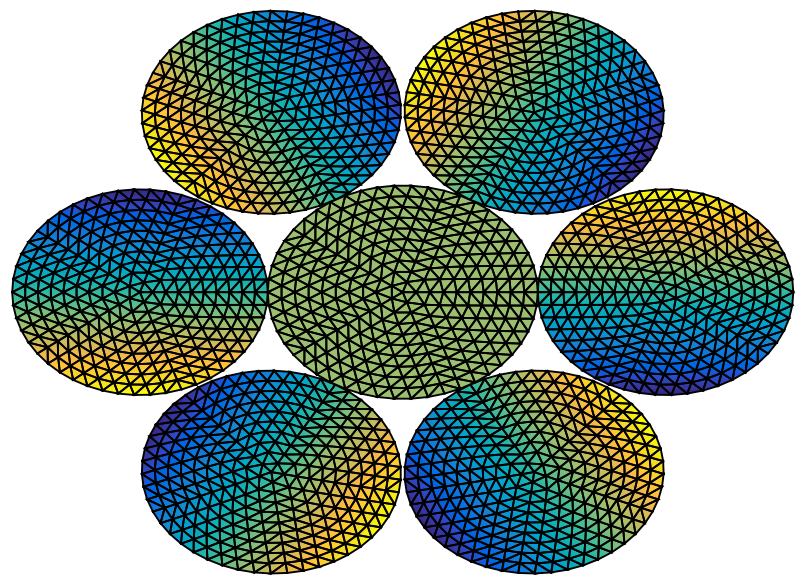

Figure 4: Single layer helical strand 


\section{Computational aspects}

The computational complexity of finite element models is a critical parameter for the analysis of large structural systems that commonly require a significant amount of degrees of freedom. Complexity assessment determines whether a task can be solved using available computational power while it provides an estimate of the time demanded. The asymptotic complexity of a linear finite element algorithm - noted as $\mathbf{O}$ - when lower order contributions are neglected, converges to [27]:

$$
\mathbf{O}_{F E M}=\mathbf{O}\left(N W^{2}\right)
$$

where $N$ stands for the number of nodes and $W$ for the bandwidth of the discrete model following a direct solution method. Elaborating on the simplified expression of Eq. 25, we can deduce a rough estimate of the relevant computational power requirements for a volumetric $(v)$ and a planar $(p l)$ modeling approach. To that extent, we consider a helical body that is discretized along its length with $n$ prismatic elements, connected with identical cross sections. For a total of $N_{h}$ helical bodies modeled, we retrieve:

$$
\mathbf{O}_{v}=4 n N_{h} \mathbf{O}_{p l}
$$

where the factor 4 stands for the duplication of the bandwidth that the connection of the lowest node of the one section to its adjacent one induces. Setting as unity the planar modeling approach complexity $\left(\mathbf{O}_{p l}=1\right)$, a graphical representation of the scaling factor between the two modeling approaches can be reconstructed, depicted in Fig. 5: 


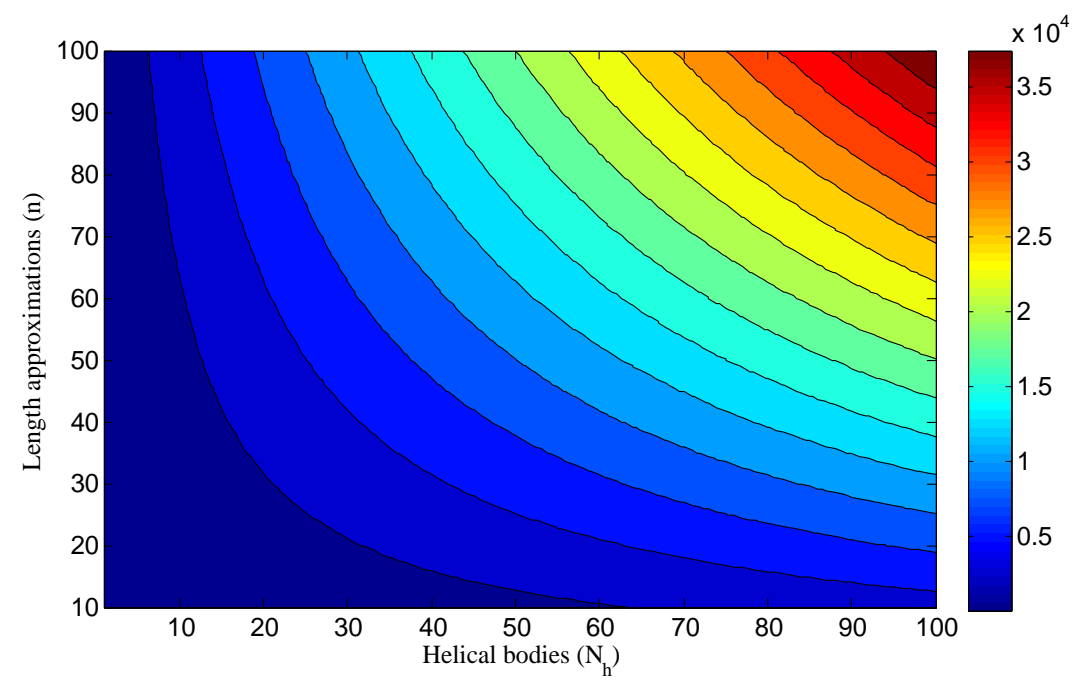

Figure 5: Computational complexity comparison

Fig. 5 suggests that the more detailed the geometric approximation $(n)$ or the larger the helical assembly $\left(N_{h}\right)$, the higher the computational resources that volumetric modeling requires, so that complexity correlation factors mounting up to orders of magnitude are retrieved, alreaddy for rather low values of the parameters involved. The latter testifies to the merit of the approach, in particular when iterative numerical patterns (Optimization, Bayesian) are to be combined, in which substantially higher values than the ones provided in Fig. 5 apply.

\section{Conclusion}

A two dimensional finite element model simulating the mechanical response of helical bodies to axial and torsional strain has been elaborated. A model verification was provided, by comparing the planar modeling numerical results to analytical closed-form stiffness expressions. Moreover, the structural response of a single layer helical strand was analyzed and confronted to established analytical and numerical models. Finally, the computational merits of the numerical approach were explicated.

\section{Acknowledgements}

The work has been supported by the Swiss National Science Foundation under project no. 200021-137544-1. 


\section{References}

[1] F.H. Hruska. Calculation of Stresses in Wire Rope. Wire and Wire Products, 26(9):766-767, 1951.

[2] F.H. Hruska. Tangential Forces in Wire Ropes. Wire and Wire Products, 28(5):455-460, 1953.

[3] K.G. McConnell and W.P. Zemek. A Model to Predict the Coupled Axial Torsion Properties of ACSR electrical Conductors. Journal of Experimental Mechanics, 22:237-244, 1982.

[4] S. Machida and A.J. Durelli. Response of a Strand to Axial and Torsional Displacements. Journal of Mechanical Engineering Science, 15:241-251, 1973.

[5] G.A. Costello. Theory of Wire Rope. Springer, New York, 1997.

[6] S.A. Velinsky. General Nonlinear Theory for Complex Wire Rope. International Journal of Mechanical Science, 27(7/8):497-507, 1985.

[7] M. Raoof and I. Kraincanic. Critical examination of various approaches used for analysing helical cables. The Journal of Strain Analysis for Engineering Design, 29(1):43-55, January 1994.

[8] W.S. Utting and N. Jones. The response of wire rope strands to axial tensile loadsPart I. Experimental results and theoretical predictions. International Journal of Mechanical Sciences, 29(9):605-619, January 1987.

[9] W.S. Utting and N.Jones. The Response of Wire Rope Strands to Axial Tensile Loads-Part II: Comparison of Experimental Results and Theoretical Predictions. International Journal of Mechanical Science, 29(9):621-636, 1987.

[10] S. Sathikh, M.B.K. Moorthy, and M. Krishnan. A symmetric linear elastic model for helical wire strands under axisymmetric loads. The Journal of Strain Analysis for Engineering Design, 31(5):389-399, September 1996.

[11] H. Ramsey. A theory of thin rods with application to helical constituent wires in cables. International Journal of Mechanical Sciences, 30(8):559570, January 1988.

[12] W.G. Jiang and M.S. Yao and J.M. Walton. A Concise Finite Element Model for Simple Straight Wire Rope Strand. International Journal of Mechanical Sciences, 41:143-161, 1999.

[13] W.G. Jiang and J.L. Henshall. The Development and Applications of the Helically Symmetric Boundary Conditions in Finite Element Analysis. Communications in Numerical Methods in Engineering, 15:435-443, 1999. 
[14] W.G. Jiang and J.L. Henshall. A Novel Finite Element Model for Helical Springs. Finite Elements in Analysis and Design, 35:363-377, 2000.

[15] A. Nawrocki and M. Labrosse. A Finite Element Model for Simple Straight Wire Rope Strands. Computers and Structures, 77:345-359, 2000.

[16] Patrice Cartraud and Tanguy Messager. Computational homogenization of periodic beam-like structures. International Journal of Solids and Structures, 43(3-4):686-696, February 2006.

[17] T. Messager and P. Cartraud. Homogenization of helical beam-like structures: application to single-walled carbon nanotubes. Computational Mechanics, 41(2):335-346, June 2007.

[18] H. Usabiaga and J.M. Pagaldays. Analytical procedure for modelling recursively and wire by wire stranded ropes subjected to traction and torsion loads. International Journal of Solids and Structures, 45(21):5503-5520, October 2008.

[19] S.R. Ghoreishi, T. Messager, P. Cartraud, and P. Davies. Validity and limitations of linear analytical models for steel wire strands under axial loading, using a 3D FE model. International Journal of Mechanical Sciences, 49(11):1251-1261, November 2007.

[20] Nikolaos Karathanasopoulos. Torsional stiffness bounds of helical structures under the influence of kinematic constraints. Structures, 3:244-249, 2015.

[21] Fabien Treyssède. Elastic waves in helical waveguides. Wave Motion, 45(4):457-470, 2008.

[22] A. Frikha, P. Cartraud, and F. Treyssède. Mechanical modeling of helical structures accounting for translational invariance. Part 1: Static behavior. International Journal of Solids and Structures, 50(9):1373-1382, May 2013.

[23] N. Karathanasopoulos and G. Kress. "A Planar Finite Element Model For Axial Strain of Helical Structures". In B.H.V. Topping, P. Iványi, (Editors), "Proceedings of the Twelfth International Conference on Computational Structures Technology", Civil-Comp Press, Stirlingshire, UK, Paper 202, 2014. doi:10.4203/ccp.106.202.

[24] M. Itskov. Tensor Algebra and Tensor Analysis for Engineers. Springer, 2007.

[25] G. Wempner. Mechanincs of solids with Applications to Thin Bodies. New York : McGraw-Hill, [1973], 1981.

[26] D. Chapelle and K.J. Bathe. The finite element analysis of shells: fundamentals. Springer, 2011.

[27] T.H. Cormen, C.E. Leiserson, R.L. Rivest, and C. Stein. Introduction To Algorithms. MIT Press, 2001. 